\title{
MODELLING A CURRENT-PROGRAMMED BUCK REGULATOR
}

\author{
R. D. MIDDLEBROOK \\ Power Electronics Group \\ Californio Institute of Technology
}

ABSTRACT

A general small-signal model for currentprogrammed switching power stages is used for design-oriented analysis of a $150 \mathrm{~W}$ buck regulator.

The model, into which the current-programming minor feedback loop is absorbed, exposes the desired tendency towards "constant" output current. The regulator voltage loop remains the only explicit feedback loop, allowing the regulator closed-loop properties to be easily obtained from those of the open-loop current-programmed power stage.

The design-oriented analytic results allow easy inference of the effects of element changes on the regulator performance functions. Results are obtained for the regulator line-to-output transfer function (audio susceptibility) and output impedance.

\section{INTRODUCTION}

Current-programmed switching power stages are becoming widely used in the power supply field because of several advantages they exhibit over conventional duty ratio programmed power stages $[1,2]$. Design of switching regulators from the control loop aspect is now well understood: smallsignal models for the power stage, and their use in design of the feedback loop, have gradually become familiar tools for design engineers over the last fifteen years.

These well-known models and methods, however, are for duty ratio programmed power stages. Corresponding models for current-programmed power stages have appeared much more recently [3,4], and have been the subject of some controversy $[5,6]$.

The purpose of this paper is to apply a particular small-signal model [6] of a currentprogrammed power stage to the anlaysis and design of a $150 \mathrm{~W}$ buck regulator. This is intended as an illustration of how the generalized model,

This work was conducted in part under the Power Electronics Program supported by a grant from GTE Communication Systems Corp., and under a contract from IBM Tucson. established once and for all, can be applied not only to the analysis but also to the design of practical regulators.

Although the loop gain is of central importance, a regulator is not specified directly by its loop gain, but by its performance functions such as output impedance and line-to-output transfer function (audio susceptibility). Therefore, the objective of the analysis is to determine how the various component values affect these functions. Understanding of the physical significance of each analytic step is essential in choosing the numerous trade-offs that have to be made. This process and its objective, of course, are applicable to any design, and are central to the approach described as design-oriented analysis.

Most of the numbers in the example $150 \mathrm{~W}$ buck regulator are the same as those chosen by Schoneman and Mitchell [7]. This was done so that the performance functions determined here could be compared with those derived by Schoneman and Mitche11, who used an entirely different approach to the analysis. When all the numbers are the same, the performance functions obtained by the two approaches are, of course, also the same. In the example discussed here, however, one parameter is chosen differently (the gain of the error amplifier) to illustrate its effect on the results.

The reasons leading to the choice of modelling approach are discussed in Section 2, and result in adoption of a canonical model that absorbs the current-programming minor feedback loop. In Section 3, this y-parameter canonical model is modified and simplified to apply specifically to the buck power stage of the example regulator. From this model, the power-stage control-to-output and line-to-output transfer functions and output impedance are immediately written down, and some comments are made regarding the effectiveness of the current-programming property.

In section 4 , the regulator voltage loop, which is now the only explicit feedback loop, is closed and the loop gain $T$ and feedback factor $1+T$ are determined. The familiar properties of singleloop feedback systems are then employed to find the regulator closed-loop output impedance $Z_{o f}$ and line-to-output transfer function $A_{\mathbf{g}}$ from their respective open-loop values. 
Also in Section 4, the results for the regulator closed-loop properties are evaluated.

Discussion of the results and of the significance of the design-oriented analysis approach is presented in Section 5 .

\section{MODELLING APPROACH}

The circuit of the regulator with a currentprogrammed buck power stage is shown in Fig. 1. The numbers, supplied to the author by private communication, are those used in an example by Schoneman and Mitchell [7].

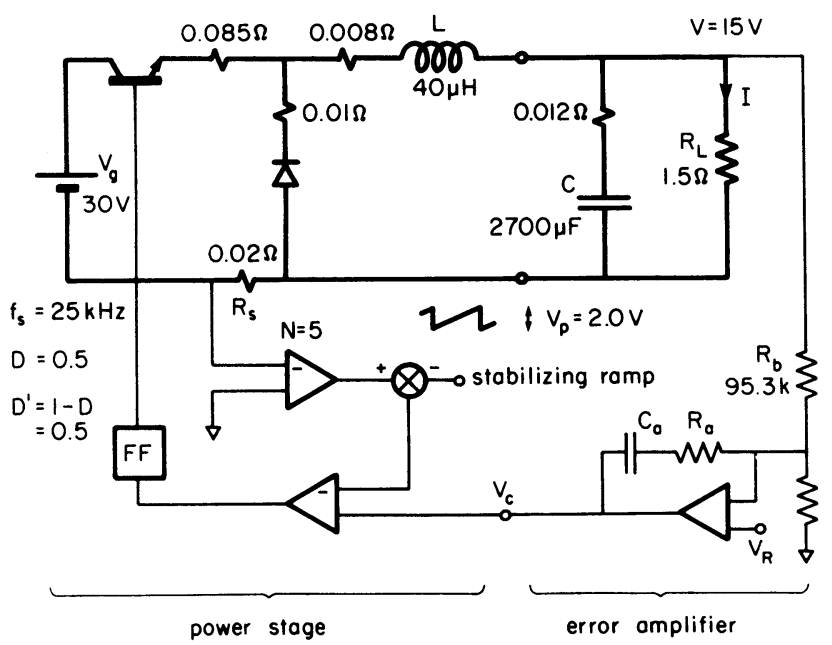

Fig. 1. Current-programmed buck regulator. Values of $R_{\mathrm{a}}$ and $C_{\mathrm{a}}$ are later chosen to be $270 \mathrm{k}$ and $0.053 \mu \mathrm{F}$.

The buck power stage and the error amplifier with gain controlled by local feedback are conventional. The reference voltage $V_{R}$ is such that the steady state duty ratio is $D=0.5$, which results in an output voltage $\mathrm{V}=15 \mathrm{~V}$ from a line voltage $V_{g}=30 \mathrm{~V}$. With a load resistance $R_{L}=1.5 \Omega$, the output current is $I=10 \mathrm{~A}$ for an output power of 150W. The switching frequency is $f_{s}-\omega_{s} / 2 \pi=$ $25 \mathrm{kHz}$, for a switching period $\mathrm{T}_{\mathrm{s}}=1 / \mathrm{f}_{\mathrm{s}}=40 \mu \mathrm{s}$.

Current-programming is implemented by $R_{s}$, which senses the switch current in the $O N$ condition. The resulting sensed voltage is multiplied by $N$, and a stabilizing ramp of peak value $2.0 \mathrm{~V}$ is added before comparison with the control signal from the error amplifier. The operation of the current-programming feature and the purpose of the stabilizing ramp have been well documented $[1,6]$.

\subsection{Choice of Feedback Loops}

Current-programming establishes a local "minor" current feedback loop around the power stage, which is inside the "major" voltage feedback loop via the error amplifier. One may define and analyze any loops or combinations of loops one wishes, each of which has its own "crossover frequency" and "phase margin" [6]; the results for the regulator performance functions should of course be the same regardless of the loop definitions.

One choice is to consider the current and voltage loops to be in parallel around the power stage $[4,5]$; this has the advantage that the familiar state-space averaged canonical model of the power stage under duty ratio programming [8] can be employed, but the disadvantage that the distinction between the current and voltage loops is lost. Since the two loops are employed for different purposes, loss of separation obscures the design criteria.

Another choice is to consider the current loop separately, and to find a canonical model that represents overall transfer functions of the current-programmed power stage around which the regulator voltage loop is closed. This approach, recommended in [6], has the advantage that design and optimization of the voltage loop proceeds in the same way as for a duty ratio programmed power stage; there is merely a different canonical model for the current-programmed power stage, whose contrasting properties are already explicitly exposed in the model. Another advantage of this approach is that the analysis of the current loop is done once and for all, and represented by its canonical model; thereafter, design of any regulator requires consideration only of the voltage loop. This is the approach that will be adopted here.

Development of a generalized canonical model for the basic current-programmed buck, boost, and buck-boost power stages has been described in [6]. This becomes the starting point for the designoriented analysis for the buck regulator of Fig. 1.

\subsection{Current-Programmed Buck Converter Canonical Model}

The canonical small-signal model for current-
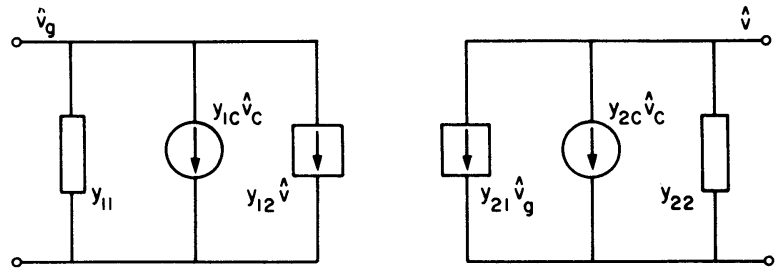

Fig. 2. Generalized small-signal y-parameter model for a current-programmed power stage. The current-programming minor feedback loop around the power stage is absorbed in, and therefore is implicit in, the model. There are two inputs, the small-signal control voltage $\hat{v}_{c}$ and line voltage $\hat{v}_{\mathrm{g}}$. 
programmed pwm converters operated in the continuous conduction mode is shown in Fig. 2 . This y-parameter model, and a table of expressions for the element values for the buck, boost, and buck-boost converters, were presented in [6].

For the buck converter, the subject of this paper, the expressions for the six elements in the model of Fig. 2 are:

$y_{12}=\frac{c_{2} D}{R} \frac{1+\frac{s D / C_{2}}{\omega_{s} / \pi}}{1+\frac{s}{\omega_{c}}} \quad y_{21}=-\frac{D\left(n D^{\prime}-1\right)}{K R} \frac{1}{1+\frac{s}{\omega_{c}}}$

$y_{1 c}=\frac{D}{R_{f}} \frac{1+\frac{s L}{R}}{1+\frac{s}{\omega_{c}}} \quad y_{2 c}=-\frac{1}{R_{f}} \frac{1}{1+\frac{s}{\omega_{c}}}$

$y_{11}=-\frac{c_{1} D^{2} 1+\frac{s / c_{1}}{\omega_{s} / \pi}}{R} \frac{n^{\prime}-D}{1+\frac{s}{\omega_{c}}} y_{22}=\frac{1}{K R} \frac{1}{1+\frac{s}{\omega_{c}}}$

where

$\mathrm{c}_{1} \equiv 1-\frac{\mathrm{nD}^{\prime}-1}{\mathrm{~K}} \quad \mathrm{c}_{2} \equiv 1-\frac{\mathrm{nD}^{\prime}-\mathrm{D}}{\mathrm{K}}$

The model of Fig. 2 and the above expressions represent the part of Fig. 1 designated the "power stage," that is, excluding the load resistance $R_{L}$ and the load capacitance $C$ and its associated esr. Also, the parasitic resistances within the power stage are neglected, which means that $R_{s}$ is accounted for solely as a current sensing device and its voltage drop in the power stage is ignored. The dc output voltage $\mathrm{V}$ in Fig. 1 is determined by two inputs, the line voltage $\mathrm{V}_{\mathrm{g}}$ and the control voltage $V_{c}$. Similarly, in the small-signal model of Fig. 2, the small-signal output voltage $\hat{v}$ is determined by the corresponding two small-signal inputs $\hat{v}_{\mathrm{g}}$ and $\hat{\mathrm{v}}_{\mathrm{c}}$.

There are several parameters in the expressions for the element values in addition to those that are explicit in Fig. 1. These are defined and evaluated below, along with a review of their significance in the operation of the currentprogrammed converter.

The current sensing function is represented by $R_{f}$, which is the ratio of the voltage presented to the comparator to the switch current (that is, the inductor current), that is being sensed. Because of the gain factor $N$ in Fig. 1 , the effective value of $R_{f}$ is $R_{f}=N R_{s}=5 \times 0.02=0.10 \Omega$.

The numerical parameter $n$ relates the equivalent current slope $M_{c}$ of the stabilizing ramp to the slope $M_{1}$ of the inductor current during the switch ON-time:

$$
\mathrm{n}=1+2 \mathrm{M}_{\mathrm{c}} / \mathrm{M}_{1}
$$

Here, the ramp reaches a peak voltage $v_{p}$ during a switching period $T_{s}$, so the equivalent current slope is $M_{c}=V_{p} / T_{s} R_{f}=2.0 /(40 \times 0.10)=0.5 \mathrm{~A} / \mu \mathrm{s}$. The inductor current slope during the switch ONtime, when the inductor is connected between line and output, is $M_{1}=\left(V_{8}-V\right) / L=15 / 40=0.375 \mathrm{~A} / \mu \mathrm{s}$. Hence, $\mathrm{n}=1+2 \times 0.5 / 0.375=3.67$.

The purpose of the stabilizing ramp is to extend the range of stability of the currentprogrammed power stage beyond the value $D_{\mathrm{max}}=0.5$ that exists in the absence of a ramp $(n=1)$. As shown in [6], in the presence of a ramp $D_{\max } /(1$ $\left.D_{\max }\right)=n$, so $D_{\max }=n /(1+n)$. In the present case $\mathrm{n}=3.67$ so $\mathrm{D}_{\max }=3.67 / 4.67=0.786$, which means that the minimum line voltage for which regulation of the output voltage $\mathrm{V}=15 \mathrm{~V}$ can be maintained is $\mathrm{V} / \mathrm{D}_{\mathrm{max}}=15 / 0.786=19.1 \mathrm{~V}$.

It is also shown in [6] that if the stabilizing ramp slope $M_{c}$ equal the slope $M_{2}$ of the inductor current during the switch OFF-time, then any disturbance from the equilibrium duty ratio is eliminated in one switching period; the value $n=n_{1}$ corresponding to $M_{c}=M_{2}$ is $n_{1}=$ $(1+D) /(1-D)=3$ for $D=0.5$. In the present buck converter example, $n=3.67$ so $M_{c}$ exceeds $M_{2}$, and in this sense the converter is "over-stabilized."

The frequency $\omega_{c}$, which appears as a pole in all six y-parameters, is given by

$$
\omega_{\mathrm{c}}=\frac{\omega_{\mathrm{s}}}{\pi \mathrm{nD}^{\prime}}-2 \pi \frac{25}{\pi \times 3.67 \times 0.5}=(2 \pi) 4.34 \mathrm{kHz}
$$

In [6], $\omega_{c}$ is identified as the crossover frequency of the loop gain of the minor current loop. This parameter represents the only visible evidence of the current feedback loop after the feedback effects have been absorbed into the y-parameter model of Fig. 2 .

The resistance $R$ is an "operating point parameter" defined as

$$
R=\frac{\text { dc output voltage }}{\text { dc output current }}=\frac{V}{I}=\frac{15}{10}=1.5 \Omega
$$

If the load on the regulator is a pure resistance $R_{L}$, as in the present example, then $R=R_{L}$ numerically. However, the distinction in symbols will be retained as a reminder that $R$ and $R_{L}$ are conceptually different; $R$ is a parameter contained in the model of the power stage, whereas $R_{L}$ is an external element. The distinction is important when the load contains a constant current component and/or becomes complex.

Finally, $\mathrm{K}$ is a "conduction parameter"

$$
\mathrm{K}=\frac{2 \mathrm{~L}}{\mathrm{RT}_{\mathrm{s}}}-\frac{2 \times 40}{1.5 \times 40}=1.33
$$

As discussed in [6], the low-frequency loop gain of the current-programmed minor feedback loop is proportional to $\mathrm{K}$, and $\mathrm{K}$ is the same conduction parameter that appears in the canonical model for a duty ratio programmed power stage in discontinuous 
conduction mode. Here, it appears in the model for a current-programmed power stage in continuous conduction mode, and must exceed a certain critical value $\mathrm{K}_{\mathrm{c} r \mathrm{t}}$ if the discontinuous conduction mode is to be avoided.

It is shown in [9] that, for a buck converter, $\mathrm{K}_{\mathrm{crit}}=\mathrm{D}^{\prime}$. In the present example of Fig. 1, D $=0.5$ so $K_{c r i t}=0.5$. From Eqs. (5) and (6), there is a corresponding minimum value $I_{m i n}$ of dc load current:

$$
I_{m i n}=\frac{V T_{s}}{2 L} K_{c \times 1 t}=\frac{15 \times 40}{2 \times 40} 0.5=3.75 \mathrm{~A}
$$

The corresponding maximum load resistance (again, for a pure resistance load), is

$$
R_{L_{\text {max }}}=R_{\max }=\frac{V}{I_{\min }}=\frac{15}{3.75}=4 \Omega
$$

If the operating conditions of Fig. 1 represent full load power of $150 \mathrm{~W}$ at $\mathrm{I}=10 \mathrm{~A}$, the minimum load power to avoid discontinuous conduction is $3.75 \mathrm{~A} \times 15 \mathrm{~V}=56 \mathrm{~W}$, or about $38 \%$ of full load power.

\section{CURRENT-PROGRAMMED BUCK REGULATOR OPEN LOOP PROPERTIES}

\subsection{Modified y-Parameter Model}

The y-parameter expressions of Eqs. (1) can be put into a somewhat more convenient form by elimination of the parameter $K$ in favor of the power stage inductance L, by Eqs. (4) and (6):

$$
\frac{\mathrm{KR}}{\mathrm{nD}}=\omega_{\mathrm{c}} \mathrm{L}
$$

For example, $\mathrm{y}_{22}$ can be written

$$
\mathrm{y}_{22}=\frac{\omega_{\mathrm{c}} \mathrm{L}}{1-\mathrm{D} / \mathrm{nD}^{\prime}} /\left(1+\frac{s}{\omega_{c}}\right)
$$

The physical interpretation of $\omega_{\mathrm{c}} \mathrm{L}=2 \pi \times 4.34 \times$ $0.04=1.09 \Omega$ is that it is the reactance of the power stage inductance at the current loop gain crossover frequency.

It follows from Eq. (10) that $1 / y_{22}$ consists of a resistance in series with an inductance. However, in the regulator circuit of $\mathrm{Fig} .11 / \mathrm{y}_{22}$, which is the output impedance of the power stage, is paralleled by $C$ and $R_{L}$. As a consequence, the inductive component of $y_{22}$ can be neglected because at frequencies where it becomes significant in $y_{22}$, the total parallel impedance is dominated by $C$. Hence (because of the way the power stage is loaded $), 1 / \mathrm{y}_{22} \approx \omega_{\mathrm{c}} \mathrm{L} /\left(1-\mathrm{D} / \mathrm{nD}^{\prime}\right)=1.09 / 0.727$ $1.50 \Omega$.

Replacement of factors in $\mathrm{K}$ by $\omega_{\mathrm{c}} \mathrm{L}$, according to Eq. (9), in the other y-parameters allows Eqs. (1) to be rewritten in the following forms: $\mathrm{y}_{12}=\frac{\mathrm{c}_{2} \mathrm{D}}{\mathrm{R}} \frac{\mathrm{sD/ \textrm {c } _ { 2 }}}{1+\frac{\mathrm{s}}{\omega_{\mathrm{c}} / \pi}} \quad \mathrm{y}_{21}=-\frac{\mathrm{D}\left(1-1 / \mathrm{nD}^{\prime}\right)}{\omega_{c} L} \frac{1}{1+\frac{s}{\omega_{c}}}$

$y_{1 c}=\frac{D}{R_{f}} \frac{1+\frac{s}{\omega_{c z}}}{1+\frac{s}{\omega_{z}}} \quad y_{2 c}=-\frac{1}{R_{f}} \frac{1}{1+\frac{s}{\omega_{c}}}$

$y_{11}=-\frac{c_{1} D^{2}}{R} \frac{1+\frac{s / c_{1}}{\omega_{s} / \pi}}{1+\frac{s}{\omega_{c}}} \quad y_{22}=\frac{1-D / n D^{\prime}}{\omega_{c} L}$

where

$c_{1}=1-\frac{\left(1-1 / n D^{\prime}\right) R}{\omega_{c} L} c_{2}=1-\frac{\left(1-D / n D^{\prime}\right) R}{\omega_{c} L}$

Figure 3 shows the small-signal model of the current-programmed boost regulator of Fig. 1, incorporating the modifications already discussed. Since the regulator input properties seen by the line are not discussed in this paper, the input half of the y-parameter model is omitted; only the output half is needed to derive the loop gain, output impedance, and line-to-output transfer function. For convenience in application of this

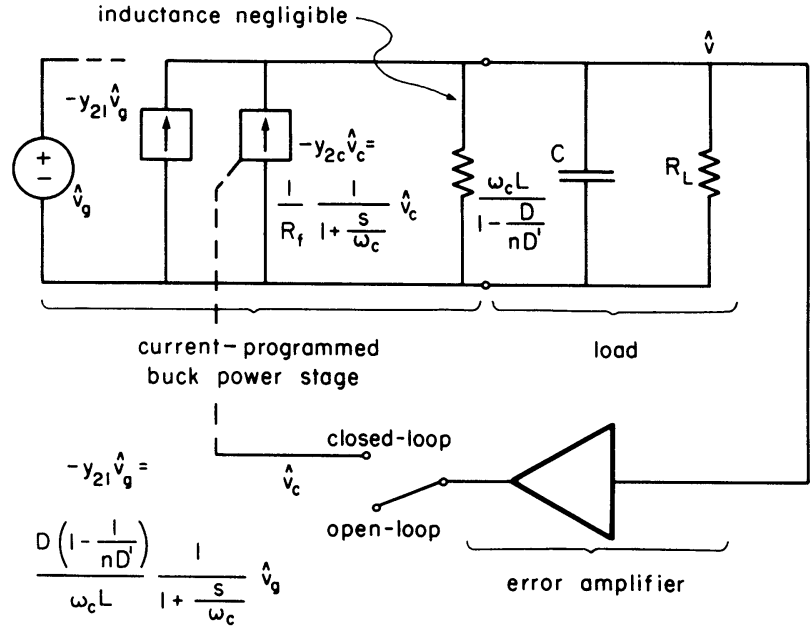

Fig. 3. Small-signal model of the circuit of Fig. 1. The y-parameter model of Fig. 2 is modified and simplified for the buck converter, and the input half is omitted. The voltage minor feedback loop around the error amplifier is absorbed in, and therefore is implicit in, the model represented by the gain block $A_{1}$. Only the voltage major feedback loop remains explicit. 
model, the numerical values already obtained are summarized below.

$$
\begin{array}{rlrl}
D & =0 . \\
C & =2700 \mu \mathrm{F} & R=R_{f} & =0.10 \Omega \\
f_{c} & =4.34 \mathrm{kHz} & R_{L} & =1.5 \Omega \\
\omega_{c} L & =1.09 \Omega
\end{array}
$$

\subsection{Open-Loop Transfer Functions $A_{c}$ $A_{8}$, and $Z_{0}$}

The switch shown in Fig. 3 is introduced merely to permit separation between the open-1oop and closed-loop properties. Three small-signal functions of the open-loop loaded power stage are of interest: the control-to-output transfer function $A_{c}=\hat{v} / \hat{v}_{c}$, the line-to-output transfer function (audio susceptibility) $A_{g}=\hat{v} / \hat{v}_{g}$, and the output impedance (including the load) $\mathrm{z}_{0}$. These functions are easily written down directly from the model of Fig. 3, and evaluated with use of Eqs. (13) through (15):

$$
A_{c}=A_{c m} \frac{1}{\left(1+s / \omega_{p}\right)\left(1+s / \omega_{c}\right)}
$$

where

$$
\begin{aligned}
A_{c m} & =\frac{\frac{\omega_{c} L}{1-D / n D^{\prime}} \| R_{L}}{R_{f}}=\frac{1.5 \| 1.5}{0.10} \\
& =7.5 \rightarrow 17.5 \mathrm{~dB} \\
\omega_{p} & =\frac{1}{\left(\frac{\omega_{c} L}{1-D / n D^{\prime}} \| R_{L}\right) C}=\frac{10^{3}}{0.75 \times 2.7} \\
& =(2 \pi) 79 \mathrm{~Hz}) \\
A_{g} & =A_{g m} \frac{1}{\left(1+s / \omega_{p}\right)\left(1+s / \omega_{c}\right)}
\end{aligned}
$$

where

$$
\begin{aligned}
A_{g m} & =\frac{D\left(1-1 / n D^{\prime}\right)}{\omega_{c} L}\left(\frac{\omega_{c} L}{1-D / n D^{\prime}} \| R_{L}\right)=0.21 \times 0.75 \\
& =0.16 \rightarrow-16 \mathrm{~dB} \\
Z_{0} & =R_{o m} \frac{1}{1+\frac{s}{\omega_{p}}}
\end{aligned}
$$

where

$$
R_{o m}=\frac{\omega_{c} L}{1-D / n D^{\prime}} \| R_{I}=0.75 \Omega \rightarrow-2.5 \mathrm{~dB} \operatorname{ref} .1 \Omega
$$

These three functions are sketched in magnitudeasymptote form in Fig. 4.

\subsection{Remarks on the Effectiveness of Current Programming}

It is worth pausing here to review the form of Fig. 3 and its significance.

In accordance with the preferred approach outlined in Section 2.1 , the regulator voltage feedback loop is explicitly exposed, while

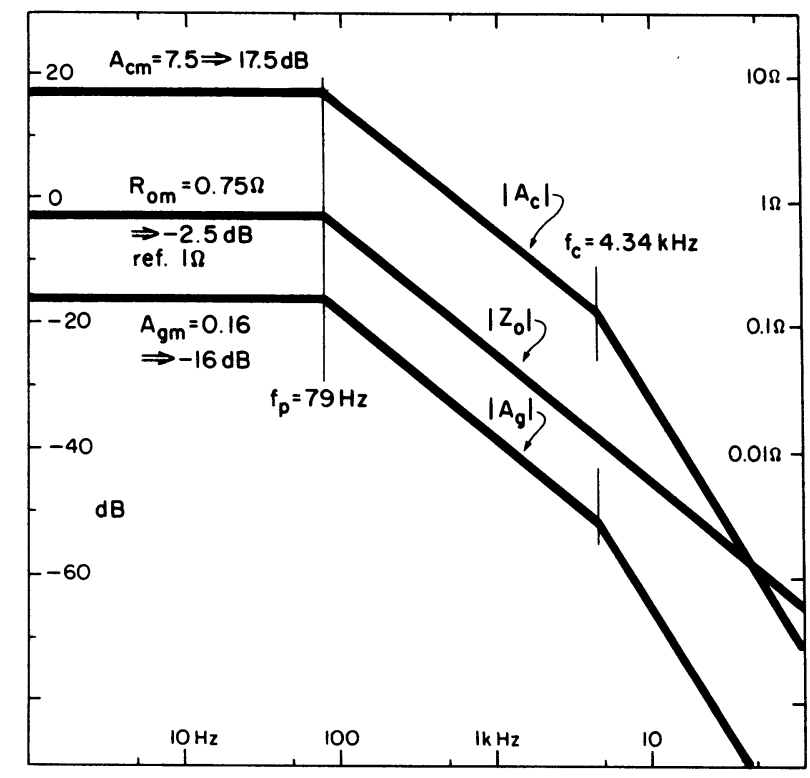

Fig. 4. Magnitude vs. frequency asymptotes of the open-loop loaded power stage transfer functions control-to-output $A_{\mathrm{c}}$ $\equiv \hat{v} / \hat{v}_{\mathrm{c}}$, line-to-output $A_{\mathrm{g}} \mathbf{\pm}$ $\hat{v} / \hat{v}_{\mathrm{g}}$, and output impedance $Z_{\mathrm{o}}$, from the model of Fig. 3 .

the current feedback loop has been absorbed into the model representing the overall properties of the current-programmed power stage. One of the advantages of this form is that the properties of the current-programmed power stage are immediately visible, which is why the three functions $A_{c}, A_{g}$, and $Z_{\circ}$ could be written directly by inspection of the model.

Also, it can be seen how well the current programming does its intended job of making the output current "constant." What this objective really means is that the output current should be represented in the model by a current generator proportional to the control voltage $\hat{v}_{c}$. It is seen that such a current generator (the $\mathrm{y}_{2 \mathrm{c}}$ generator) is indeed present, but that the output current is not equal to it because the output resistance is not infinite. The output resistance is $\omega_{c} \mathrm{~L} /\left(1-\mathrm{D} / \mathrm{nD}^{\prime}\right)$ and, as shown in [6], the denominator only goes to zero in the limit of instability when $n \rightarrow D / D^{\prime}$. Therefore, the output resistance can only go to infinity if either the switching frequency or the inductance goes to infinity, a limit that corresponds to infinite loop gain of the current minor feedback loop [6]. The actual value of the output resistance $\omega_{c} L /(1$ $\left.\mathrm{D} / \mathrm{nD}^{\prime}\right)$, in the present example, is $1.5 \Omega$, and not at all large, consistent with the point made in [6] that the low-frequency current loop gain is not large.

Finally, the current generator $y_{2 c} \hat{v}_{c}$ itself in Fig. 3 fails to "program" its current at higher frequencies, because of the pole $\omega_{c}$. As already mentioned, this pole is the crossover frequency of the current loop gain, that is, the frequency above which the current-programming ceases to function at 
al1. Consequently, the control-to-output transfer function $A_{c}$ given by Eq. (16) contains not only the dominant pole $\omega_{\mathrm{p}}$ (commonly thought to be the "only corner frequency" in this function), but also a second pole $\omega_{c}$, as shown graphically in Fig. 4.

Were it not for these "shortcomings" of the current feedback loop, the $\mathrm{y}_{2 \mathrm{c}} \hat{\mathrm{v}}_{\mathrm{c}}$ current generator in Fig. 3 would be $1 / R_{f}$, and this would also be the output current, so that the output current would ideally be programmed to be equal to $\left(1 / R_{f}\right) \hat{v}_{c}$.

Thus $R_{f}$, the effective current sense resistance, sets the control voltage to output current gain, and therefore appears as an explicit (reciprocal) factor in the control voltage to output voltage transfer function given by Eqs. (16) and (17). This transfer function implicitly applies to the power stage with the minor currentprogramming feedback loop closed, just as the gain block labeled $A_{1}$ in Fig. 3 applies to the error amplifier with its minor voltage feedback loop closed.

In this way, the various feedback loops are accounted for in an orderly fashion, and in the model of Fig. 3 only the regulator major voltage feedback loop remains to be considered. This is done in the familiar manner used for conventional "single-loop" regulators, the only difference being the incorporation of a different canonical model for the power stage.

\section{CURRENT-PROGRAYMED BUCK REGULATOR CLOSED-LOOP} PROPERTIES

The model of Fig. 3 contains all the information necessary for establishment of the major voltage loop gain and the regulator closedloop output impedance $Z_{o f}$ and line-to-output transfer function $A_{\mathbf{g} f}$.

\subsection{Loop Gain $T$, Phase Margin $\phi_{M}$ and Feedback Factor $1+T$}

The voltage loop gain $T$ is simply the product of the error amplifier gain $A_{1}$ and the control-tooutput transfer function $A_{c}$ of the loaded power stage:

$$
T=A_{1} A_{c}
$$

If $A_{1}$ is a constant $A_{1 m}$, the loop gain is merely a vertical scaling of the function $A_{c}$ with its various corner frequencies. This is the same as saying that the value of $A_{1 m}$ determines not only the midband loop gain $\mathrm{T}_{\mathrm{m}}$ as

$$
T_{m}=A_{1 m} A_{c m}
$$

but also the loop gain crossover frequency $f_{v c}=$ $\omega_{\mathrm{vc}} / 2 \pi$, the frequency where the magnitude of $\mathrm{T}$ crosses the zero $\mathrm{dB}$ axis, as shown in Fig. 5. By the geometry of the graph,

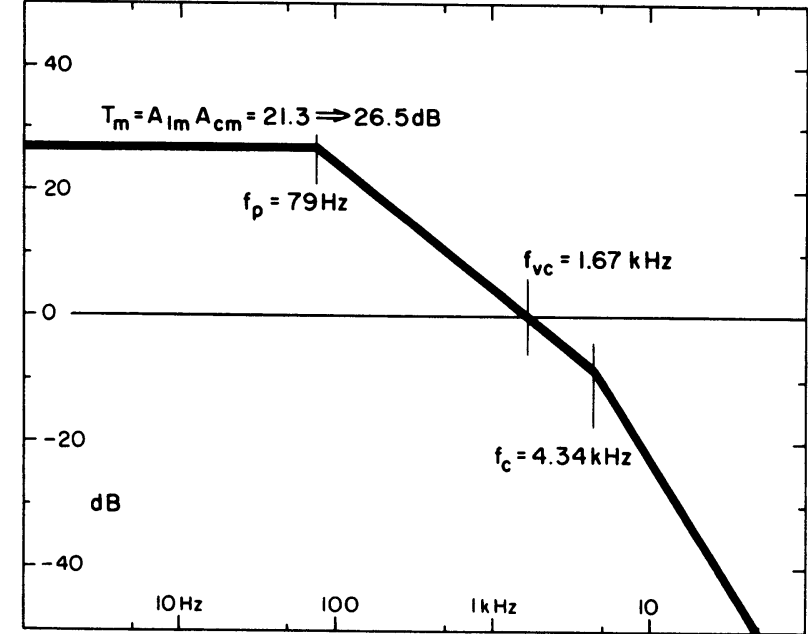

Fig. 5. Placement of the loop gain crossover frequency $f_{\mathrm{vc}}$ at $3.43 \mathrm{kHz}$, by appropriate choice of the midband loop gain $T_{\mathrm{m}}$, and hence of the error amplifier gain $A_{1 \mathrm{~m}}$.

$$
\omega_{\mathrm{vc}}=\mathrm{T}_{\mathrm{m}} \omega_{\mathrm{p}}
$$

The value of $A_{1 m}$ must be chosen so that crossover occurs below the pole $\omega_{c}$, because otherwise the phase margin would be too small. Obviously, one wishes to place the crossover frequency as high as possible in order to get the widest bandwidth over which the benefits of feedback are realized.

Where shall the loop gain crossover frequency $f_{v c}$ be placed? Let us try putting $f_{v c}$ at about one-third of the pole frequency $f_{c}=3.34 \mathrm{kHz}$, say $f_{v c}=1.67 \mathrm{kHz}$

The resulting phase margin $\phi_{M}$ of the voltage loop gain is $180^{\circ}$ minus the sum of the lag contributions from the poles at $f_{p}$ and $f_{c}$ :

$$
\begin{aligned}
\phi_{M} & =180-\left(\tan ^{-1} \frac{1670}{79}+\tan ^{-1} \frac{1.67}{4.34}\right) \\
& =180-\left(87^{\circ}+21^{\circ}\right)=72^{\circ}
\end{aligned}
$$

This is an acceptable phase margin, so we can adopt the crossover frequency $f_{v c}=1.67 \mathrm{kHz}$, and use Eq. (25) to find the corresponding midband loop gain as $\mathrm{T}_{\mathrm{m}}=\mathrm{f}_{\mathrm{vc}} / \mathrm{f}_{\mathrm{p}}=1670 / 79-21.3 \rightarrow 26.5 \mathrm{~dB}$. From Eq. (24), the required value of the error amplifier gain is $A_{1 m}=T_{m} / A_{c m}=21.3 / 7.5=2.84 \rightarrow 9.1 \mathrm{~dB}$.

The error amplifier is actually an opamp with local feedback to set its overall gain $A_{1}$, and hence the crossover frequency of the regulator major voltage loop. Since only $9.1 \mathrm{~dB}$ of gain is required, most of the available opamp gain is wasted. However, the lost gain can be recovered, for frequencies sufficiently far below the regulator loop crossover frequency, by placing an "inverted zero" $\omega_{1}$ in the error amplifier gain function: 


$$
A_{1}=A_{1 m}\left(1+\frac{\omega_{1}}{s}\right)
$$

From the circuit in Fig. 1 ,

$$
\begin{aligned}
& A_{1 \mathrm{~m}}=\mathrm{R}_{\mathrm{a}} / \mathrm{R}_{\mathrm{b}} \\
& \omega_{1}=\frac{1}{\mathrm{C}_{\mathrm{a}} \mathrm{R}_{\mathrm{a}}}
\end{aligned}
$$

The zero $\omega_{1}$ is open to choice; let us make $\omega_{1} / 2 \pi=$ $f_{1}=11 \mathrm{~Hz}$. Since $A_{1 \mathrm{~m}}$ is already set at 2.84 and $R_{b}$ -95.3k is specified, the required values of $R_{a}$ and $\mathrm{C}_{\mathrm{a}}$ are:

$$
\begin{aligned}
& \mathrm{R}_{\mathrm{a}}=\mathrm{A}_{1 \mathrm{~m}} \mathrm{R}_{\mathrm{b}}=2.84 \times 95.3=270 \mathrm{k} \\
& \mathrm{C}_{\mathrm{a}}=\frac{1}{\omega_{1} \mathrm{R}_{\mathrm{a}}}=\frac{10^{-3}}{2 \pi \times 11 \times 270}=0.053 \mu \mathrm{F}
\end{aligned}
$$

The final designed loop gain $T$ is shown in Fig. 6. The inverted zero at $f_{1}=11 \mathrm{~Hz}$ contributes a small additional phase lag $\tan ^{-1}(11 / 1670)=0.4^{\circ}$ at the crossover frequency $\mathrm{f}_{\mathrm{vc}}=1.67 \mathrm{kHz}$, resulting in a negligible reduction in the original phase margin $\phi_{M}=72^{\circ}$.

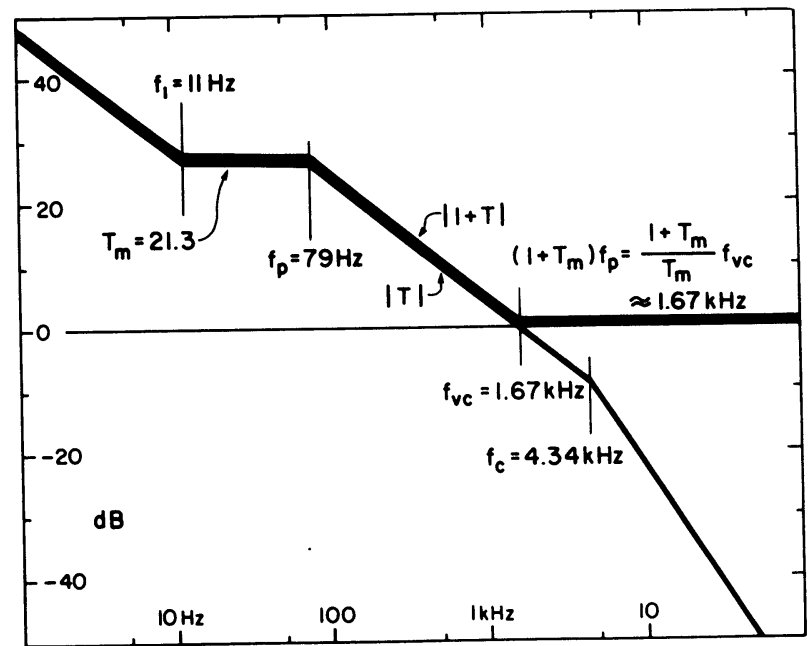

Fig. 6. Construction of the feedback factor $1+T$ from the loop gain $T$, after the inverted zero chosen to be at $f_{1}=11 \mathrm{~Hz}$ has been introduced into $T$, for minimum load resistance $R_{\mathrm{L}}=R=1.5 \Omega$.

The expression for the final regulator voltage loop gain, by incorporation of Eqs. and (27) into (23), is

$$
T=T_{m} \frac{1+\frac{\omega_{1}}{s}}{\left(1+\frac{s}{\omega_{p}}\right)\left(1+\frac{s}{\omega_{c}}\right)}
$$

where

$$
\begin{aligned}
T_{m} & =A_{1 m} A_{c m}=A_{1 m} \frac{\frac{\omega_{c} L}{1-D / n D}, \| R_{L}}{R_{f}} \\
& =21.3 \rightarrow 26.5 \mathrm{~dB}
\end{aligned}
$$

Also, the regulator voltage loop crossover frequency is, from Eqs. (18) and (25),

$$
\begin{aligned}
\omega_{v c} & =T_{m} \omega_{p}=\frac{A_{1 m}}{R_{f c}} \\
& =\frac{2.84 \times 10^{6}}{0.10 \times 2700}=(2 \pi) 1.67 \mathrm{kHz}
\end{aligned}
$$

The above numerical results for $T_{m}$ and $\omega_{v m}$ of course confirm the values that led to choice of $A_{1 m}$ in the first place. To complete the summary of analytical and numerical results, the expressions and numbers for the corner frequencies are repeated below:

$$
\begin{aligned}
& \omega_{\mathrm{p}}=\frac{1}{\left(\frac{\omega_{\mathrm{c}} \mathrm{L}}{1-\mathrm{D} / \mathrm{nD^{ \prime }}} \| \mathrm{R}_{\mathrm{L}}\right)_{\mathrm{C}}}=(2 \pi) 79 \mathrm{~Hz} \\
& \omega_{\mathrm{c}}=\frac{\omega_{\mathrm{s}}}{\pi n \mathrm{nD}^{\prime}}-(2 \pi) 4.34 \mathrm{kHz}
\end{aligned}
$$

One of the most important advantages of the adopted modelling approach is that the properties of the current-programmed power stage are already explicit before the regulator major voltage loop is considered. The direct result of this approach is that the regulator closed-loop properties can be found from the open-loop properties by the familiar formulas for single-1oop systems.

In particular, the regulator closed-loop output impedance $\mathrm{Z}_{\mathrm{of}}$ and line-to-output transfer functions $A_{g} f$ are given in terms of their respective open-loop values $Z_{o}$ and $A_{g}$ by

$$
\begin{aligned}
& Z_{o f}=\frac{Z_{o}}{1+T} \\
& A_{g f}=\frac{A_{g}}{1+T}
\end{aligned}
$$

where $T$ is the regulator major voltage loop gain given by Eqs. (32) through (38).

Only one additional calculation is required, which is to find the feedback factor $1+T$ from the known $T$. This can be done very easily, to a sufficient degree of accuracy, by a semigraphical technique.

As shown in Fig. $6,1+\mathrm{T}$ can be constructed by drawing the asymptotes for $1+T$ just above those of $T$ for frequencies below the crossover $f_{v c}$. However, beyond crossover, $T$ is much less than unity, so $1+T \approx 1$ or $0 \mathrm{~dB}$. All that remains is to identify the corner frequencies of $1+T$. The inverted zero $f_{1}$ and the lowest pole $f_{p}$ are the same as those in $\mathrm{T}$; the new zero, by the geometry of the asymptotes, is $\left(1+T_{m}\right) f_{p}$ or $\left(1+T_{m}\right) f_{v c} / T_{m}$. Hence, the factored pole-zero expression for $1+T$ can be written by inspection of the asymptotes as

$$
1+T=\left(1+T_{m}\right) \frac{\left(1+\frac{\omega_{1}}{s}\right)\left(1+\frac{T_{m} s}{\left(1+T_{m}\right) \omega_{v c}}\right)}{\left(1+\frac{s}{\omega_{p}}\right)}
$$


It is to be noted that if Eq. (32) for $T$ were substituted into $1+T$ and the pole-zero factors found algebraically, a cubic equation would have to be solved. With appropriate approximation, the result of Eq. (41) would be obtained.

\subsection{Closed-Loop Transfer Functions $Z_{\text {of }}$ and $A_{g} f$ for Maximum Load}

The regulator closed-loop output impedance $Z_{\text {of }}$ can now be found from substitution of Eqs. (21) and (41) into (39):

$$
Z_{o f}=R_{o f m} \frac{1}{\left(1+\frac{\omega_{1}}{s}\right)\left(1+\frac{T_{m} s}{\left(1+T_{m}\right) \omega_{v c}}\right)}
$$

where

$$
\begin{aligned}
R_{o f m} & \equiv \frac{R_{o m}}{1+T_{m}}=\frac{T_{m}}{1+T_{m}} \frac{R_{o m}}{T_{m}} \\
& =\frac{T_{m}}{1+T_{m}} \frac{R_{f}}{A_{1 m}}=\frac{T_{m}}{1+T_{m}} \frac{1}{\omega_{v c} C} \\
& =\frac{21.3}{22.3} \frac{0.10}{2.84}=0.034 \Omega
\end{aligned}
$$

Although the algebra is simple, more insight is gained into the above result if the process is conducted graphically. In Fig. 7, the magnitude asymptotes for $Z_{0}$ and $1+T$ are shown; their difference gives $Z_{o} /(1+T)=Z_{o f}$.

The same graphical process can be used to find the regulator closed-loop line-to-output

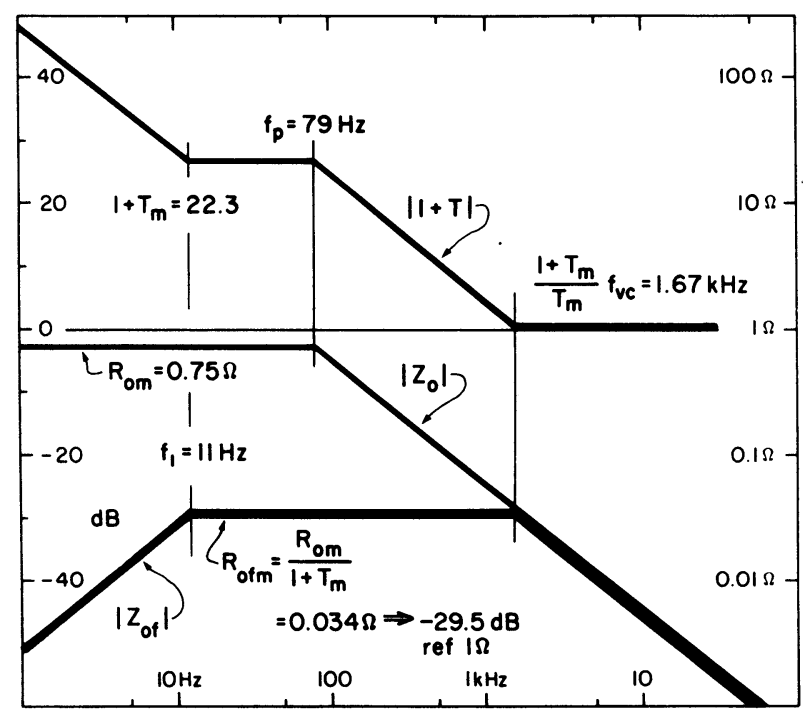

Fig. 7. Construction of the closed-loop output impedance $Z_{\text {of }}$ from its open-loop value $Z_{0}$ and the feedback factor $1+T$, according to $Z_{\mathrm{Of}}=Z_{\mathrm{o}} /(1+T)$, for $R_{\mathrm{L}}=R$ $=1.5 \Omega$.

transfer function. Figure 8 shows the magnitude asymptotes of $A_{g}$ and $1+T$ from Eqs. (19) and (41); their difference gives $A_{g} /(1+T)=A_{g}$. Analytically, the result is

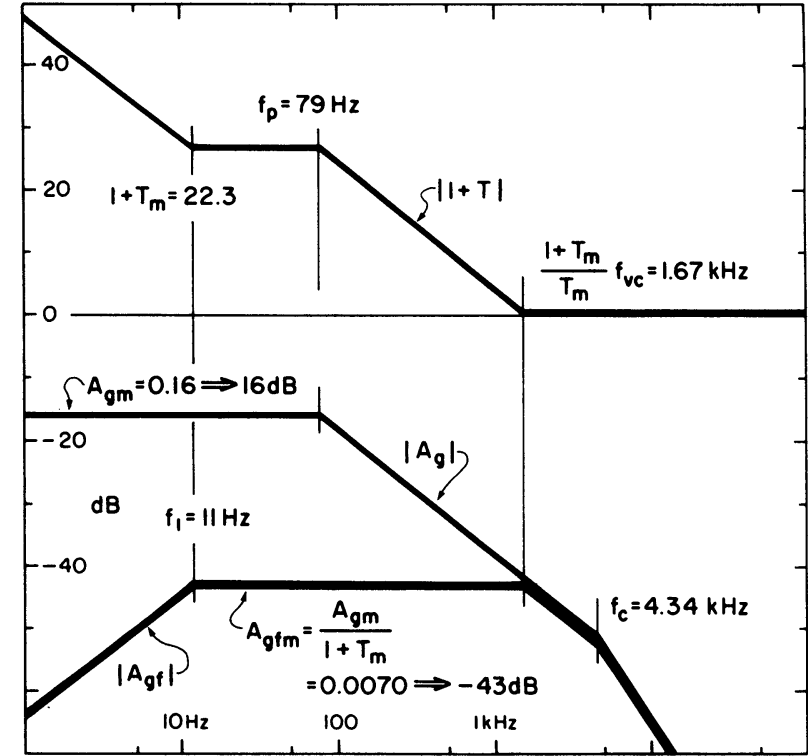

Fig. 8. Construction of the closed-loop line-tooutput transfer function $A_{\mathrm{g} f}$ from its open-loop value $A_{\mathrm{g}}$ and the feedback factor $1+T$, according to $A_{\mathrm{g} f}=A_{\mathrm{g}} /(1+T)$ for $R_{\mathrm{L}}=R=1.5 \Omega$.

$$
A_{g f}=\dot{A}_{g f m} \frac{1}{\left(1+\frac{\omega_{1}}{s}\right)\left(\frac{T_{m} s}{\left(1+T_{m}\right) \omega_{v m}}\right)\left(1+\frac{s}{\omega_{c}}\right)}
$$

where

$$
\begin{aligned}
A_{g f m} & =\frac{A_{g m}}{1+T_{m}}=\frac{T_{m}}{1+T_{m}} \frac{A_{g m}}{T_{m}} \\
& =\frac{T_{m}}{1+T_{m}} \frac{D\left(1-1 / n D^{\prime}\right) R}{A_{1 m} \omega_{c} L} \\
& =\frac{T_{m}}{1+T_{m}} \frac{D\left(1-1 / n D^{\prime}\right)}{\omega_{c} L} \frac{1}{\omega_{v c} C} \\
& =\frac{21.3}{22.3} \frac{0.5 \times 0.455 \times 0.10}{2.84 \times 1.09} \\
& =0.0070 \rightarrow-43 \mathrm{~dB}
\end{aligned}
$$

\section{SIGNIFICANCE AND INTERPRETATION OF THE DESIGN-ORIENTED ANALYSIS}

Analysis is only one of the tools used in design, and analysis is only useful if its results can be used in reverse to select element values in order to achieve certain performance results, namely, to meet the specifications.

The interpretation of the analytic results obtained above, discussed in this section, is indispensable if full value is to be obtained from the analysis. This is, after all, the whole purpose of the "design-oriented analysis" approach.

The most important aspects of the analytic results are the loop gain crossover frequency and the midband (maximum) values of the closed-loop 
output impedance and line-to-output transfer function, and how they vary from minimum to maximum load resistance, and from minimum to maximum line voltage. The load resistance $R_{L}$ enters the equations directly, and also indirectly through the operating point parameter $R$, which for a purely resistive load is numerically equal to $R_{L}$. In this example of the buck regulator, the minimum value is $R=R_{L}=1.5 \Omega$ (maximum power out of $150 \mathrm{~W}$ ), and the maximum value is $R=R_{L}=4 \Omega$ (minimum power out of $56 \mathrm{~W})$, as discussed in Section 2.2. The minimum line voltage, also discussed in section 2.2 , is 19.1V. The line voltage enters the equations through the duty ratio $D$ needed to maintain the regulated output voltage $\mathrm{V}=15 \mathrm{~V}$.

Although the midband loop gain $T_{m}$, by Eq. (33), varies quite strongly with both $D$ and $R_{L}$, the crossover frequency $\omega_{v c}=A_{1 m} / R_{f} C$, by Eq. (35), is independent of both line and load. This is because the dominant pole $\omega_{\mathrm{p}}$, Eq. (37), varies inversely with $T_{m}$ as the same function of $D$ and $R_{L}$ through the common factor $\left[\omega_{c} L /\left(1-D / n D^{\prime}\right)\right] \| R_{L}$. It is, of course, desirable to have a crossover frequency that changes little, if at all, with operating conditions.

On the other hand, the midband loop gain $T_{m}$ itself does not have any particular significance because it is explicit only over the narrow frequency range between the inverted zero $\omega_{1}$ and the dominant pole $\omega_{\mathrm{p}}$. The final pole $\omega_{\mathrm{c}}$ in the loop gain, Eq. (4), is also independent of load, but does depend on line voltage.

The feedback factor $1+T$ essentially follows $T$ until it levels out at unity at the zero $\left(1+T_{m}\right) \omega_{v c} / T_{m}$. The midband loop gain is sufficiently high that $\left(1+\mathrm{T}_{\mathrm{m}}\right) / \mathrm{T}_{\mathrm{m}} \approx 1$ and so $1+\mathrm{T}$ levels out at essentially the crossover frequency $f_{\mathrm{vc}}=4.34 \mathrm{kHz}$.

The closed-loop output impedance $z_{o f}$ is its open-loop value divided by the feedback factor, which results in Eq. (42). Since the loop gain goes away at the crossover frequency, the closedloop output impedance is essentially equal to its open-loop value beyond $\omega_{\mathrm{vc}}$; since its open-loop value is dominated by the load capacitance $C$, the midband (maximum) closed-1oop value $R_{o f m}$ is equal to the load capacitance reactance at the frequency $\left(1+T_{m}\right) \omega_{v c} / T_{m}$, as seen from Eq. (43). The closedloop output impedance drops below $R_{\text {ofm }}$ at decreasing frequencies below $\omega_{1}$ because the error amplifier gain increases, and so the loop gain increases faster than the capacitance reactance.

The most significant consequence of these points is that the closed-loop output impedance $Z_{\text {of }}$ is independent of load $R_{L}$ (and of the operating point parameter $R$ ), to the extent that $\left(1+T_{m}\right) / T_{m} \approx$ 1.

Very similar remarks may be made concerning the closed-loop line-to-output transfer function $\mathrm{A}_{\mathrm{g} f}$ of Eq. (44), which is also equal to its openloop value divided by the feedback factor. The midband (maximum) closed-loop value $A_{g f m}$ is equal to its open-loop value at the frequency
$\left(1+T_{m}\right) \omega_{v c} / T_{m}$, as seen from Eq. ( ). As in the case of the output impedance, this maximum value does not depend on the operating point parameter $R$.

Some further points can be made regarding the design choices. The central importance of the loop gain crossover frequency has been emphasized: it determines the midband value of the closed-loop output impedance and of the line-to-output transfer function, and the pole above which they fall off. Clearly, it is desirable to have as high a crossover frequency $f_{v c}$ as possible, limited by its encroachment on the second pole $\omega_{c}$ of the power stage and consequent reduction of phase margin.

The other frequency of salient importance is the error amplifier inverted zero at $f_{1}$. It is desirable to have $f_{1}$ as high as possible in order to make as narrow as possible the frequency over which the closed-loop output impedance and line-tooutput transfer function have their maximum values (Figs. 8). The limiting factor is again phase margin; a higher $f_{1}$ reduces $\phi_{M}$. The design choices of $A_{1 \mathrm{~m}}$ and $f_{1}$ are implemented by the appropriate values of $R_{a}$ and $C_{a}$ in the error amplifier circuit of Fig. 1, as given by Eqs. (30) and (31).

The consequence of too low a phase margin, whether because of proximity of either the second pole $\omega_{c}$ or the inverted zero $f_{1}$ to the crossover frequency $f_{v c}$, is that both the closed-loop output impedance and line-to-output transfer functions develop a resonant peak above their midband values. In the extreme, of course, instability results.

Consideration has been given here mainly to the effects of load resistance upon the regulator performance functions. However, all the information relevant to the current-programmed buck regulator of Fig. 1 is contained in the model of Fig. 3. A complete design should of course also take into account a range of line voltages $V_{8}$, which would be accounted for in the model by a corresponding range of duty ratio $D$.

Other choices of stabilizing ramp slope $M_{c}$, represented through the parameter $\mathrm{n}$ of $\mathrm{Eq}$. (3), could also be considered. It has already been mentioned in Section 2.2 that the power stage is "over-stabilized" in the sense that $M_{c}$ exceeds $M_{2}$, the declining inductance current slope during the power switch OFF-time. The power stage is even more over-stabilized in another sense, related to the line-to-output transfer function $\mathrm{A}_{\mathrm{g}}$.

As seen from Fig. 3 and Eq. (20), the lineto-output transfer function $A_{g}$ contains the factor $\left(1-1 / \mathrm{nD}^{\prime}\right.$ ) (from the $\mathrm{y}_{21}$ element of the $\mathrm{y}$-parameter model of the current-programmed power stage). This factor is zero if $\mathrm{nD}^{\prime}=1$. From Eq. (3), this occurs if the stabilizing ramp slope is chosen to be $M_{c}=M_{c o}$ such that

$$
M_{c \circ}=\frac{1}{2} \frac{D}{D^{\prime}} M_{1}=\frac{1}{2} M_{2}=\frac{1}{2} \frac{V}{L}
$$

In the example regulator, this corresponds to a voltage stabilizing ramp amplitude $\mathrm{V}_{\mathrm{p}}=0.76 \mathrm{~V}$, rather than the original value $\mathrm{v}_{\mathrm{p}}=2.0 \mathrm{~V}$. Moreover, this optimum value is independent of 
operating point for a given regulated output voltage.

Almost all the numbers in the example circuit of Fig. 1 are the same as those used by Schoneman and Mitchell [7], who presented experimental measurements of the closed-loop output impedance and line-to-output transfer function to verify predictions made by an entirely different modelling approach.

The only regulator design parameter in this paper chosen differently from that of Schoneman and Mitchell is the error amplifier midband gain $A_{1 m}=$ $R_{a} / R_{b}$, which is here set at $A_{1 m}=2.84$ with a required resistance $R_{a}=270 k$. The value of $C_{a}$ here is different from that of Schoneman and Mitchell only to maintain the same value of the inverted zero $f_{1}=11 \mathrm{~Hz}$.

Although the regulator properties and transfer functions derived in this paper have not been directly verified experimentally by the author, they agree completely with the experimental measurements presented by Schoneman and Mitchell when their value $A_{1 \mathrm{~m}}=78.7 / 95.3=0.83$ is employed. This may be taken to be adequate proof of the validity of the canonical model for the current-programmed boost power stage of Fig. 3 . The value $A_{1 m}=0.26$ implies a midband loop gain of only $T_{m}=7.9$, and correspondingly larger values $R_{o f m}=0.56 \Omega$ and $A_{B f m}=0.11$ at a load resistance of $11.2 \Omega$.

There is actually one other difference from the model of Schoneman and Mitchell. In the circuit of Fig. 1, the resistance $0.012 \Omega$ in series with the $2700 \mu \mathrm{F}$ capacitance gives a zero at $\omega_{2}=(2$ $\pi) 5.0 \mathrm{kHz}$ which has been ignored throughout this paper in order to eliminate the corresponding factor from numerous equations. It could easily be replaced, and would appear as a factor $\left(1+s / \omega_{2}\right)$ in the numerators of the expressions for the loop gain and the open-loop and closed-loop output impedance and line-to-output transfer functions.

Both the modelling approach employed in this paper and that of Schoneman and Mitchell are based on the state-space averaging method for switchedmode converters [8]. This means that results at frequencies approaching the switching frequency $f_{s}$ $=25 \mathrm{kHz}$ are not reliable.

\section{CONCLUSIONS}

Current-programming of switching power stages is becoming widely adopted. However, application of more recently presented equivalent circuit models has not been fully developed. This paper discusses the use of a particular model for the power stage in the analysis and design of a current-programmed boost regulator.

Since current-programming introduces a minor feedback loop around the power stage, which is inside the regulator major voltage loop, a choice must be made at the outset as to which loop or combination of loops are to be adopted for analysis purposes. The merits of alternative choices are discussed in Section 1, and additional comments are offered in [10].

The approach chosen is that in which the current-programming minor loop is absorbed into an equivalent circuit (canonical model) that represents the properties of the current-programmed power stage as a whole. The benefit gained is that the degree to which the current-programming is effective can immediately be seen explicitly from this model, which then becomes one transfer block in the model of the regulator major voltage feedback loop. Thence, the familiar methods for single-loop feedback systems can be employed. The simplicity of this approach is of significant value in the method of design-oriented analysis, in which the analytic results (which are not "answers" in themselves) can be used in reverse to make design choices and tradeoffs.

This procedure is pursued in the following sections, applied for illustration to a 150W current-programmed buck regulator switched at $25 \mathrm{kHz}$.

In Section 3, the y-parameter canonical model for a current-programmed power stage is modified and simplified for specific application to the example buck converter, and immediately leads to simple factored pole-zero expressions for the control-to-output and line-to-output (audio susceptibility) transfer functions $A_{c}$ and $A_{g}$, and for the power-stage output impedance $Z_{0}$.

These open-loop functions are used in section 4 to determine the regulator (single-) loop gain $T$ and, by a simple semigraphical technique, the feedback factor 1+T. The feedback factor in turn is used to find the regulator closed-loop properties $Z_{o f}$ and $A_{g f}$ that correspond to the converter open-loop values, by $Z_{o f}-Z_{o} /(1+T)$ and $A_{g f} /(1+T)$.

These steps represent the essence of the design-oriented analysis approach, in which the analytic results, which are to match the specifications, are in simple forms closely related to the original elements in the system. The significance and interpretation of the designoriented analysis approach as applied to the example regulator are discussed at some length in

A treatment similar to that given in this paper for a buck regulator has been presented for a current-programmed boost regulator in [11]. 
REFERENCES

[1] Cecil W. Deisch, "Simple Switching Control Method Changes Power Converter into a Current Source," IEEE Power Electronics Specialists Conference, 1978 Record, pp. 300 - 306 (IEEE Publication 78CH1337-5 AES).

[2] A. Cape1, G. Ferrante, D. O'Sullivan, and A. Weinberg, "Application of the Injected Current Model for the Dynamic Analysis of Switching Regulators with the New Concept of LC $^{3}$ Modulator," IEEE Power Electronics Specialists Conference, 1978 Record, pp. 135 - 147 (IEEE Publication 78CH1337-5 AES).

[3] Shi-Ping Hsu, Art Brown, Loman Rensink, and R. D. Middlebrook, "Modelling and Analysis of Switching Dc-to-Dc Converters in ConstantFrequency Current-Programmed Mode," IEEE Power Electronics Specialists Conference, 1979 Record, pp. 284 - 301 (IEEE Publication 79CH1461-3 AES).

[4] Daniel M. Mitche11, "An Analytical Investigation of Current-Injected Control for Constant-Frequency Switching Regulators," 1985 IEEE Power Electronics Specialists Conference, Proceedings of ESA Sessions, pp. 225 - 233 (ESA Publication SP-230).

[5] B. H. Cho and F. C. Lee, "Measurement of Loop Gain with the Digital Modulator," IEEE Power Electronics Specialists Conference, 1984 Record, pp. 363 - 373 (IEEE Publication $84 \mathrm{CH} 2000-8$ ).

[6] R. D. Middlebrook, "Topics in Multiple-Loop Regulators and Current-Mode Programming," IEEE Power Electronics Specialists Conference, 1985 Record, pp. 716 - 732 (IEEE Publication 85CH2117-0).

[7] George K. Schoneman and Daniel M. Mitche11, "Closed-Loop Performance Comparisons of Switching Regulators with Current Injected Control," IEEE Power Electronics Specialists Conference, 1986 Record, pp. 3 - 12 (IEEE Publication 86CH2310-1).
[8] R. D. Middlebrook and Slobodan Ćuk, "A General Unified Approach to Modelling Switching Converter Power Stages," IEEE Power Electronics Specialists Conference, 1976 Record, pp. 18 - 34 (IEEE Publication 76CH1084-3 AES); also International J. of Electronics, vol. 42, no. 6, pp. $521-550$, June 1977.

[9] Slobodan Cuk and R. D. Middlebrook, "A General Unified Approach to Modelling Switching Dc-to-Dc Converters in Discontinuous Conduction Mode," IEEE Power Electronics Specialists Conference, 1977 Record, pp. 36 - 57 (IEEE Publication 77CH1213-8 AES).

[10] R. B. Ridley, B. H. Cho, and F. C. Lee, "Analysis and Interpretation of Loop Gain Measurements of Multi-Loop Controlled Switching Regulators," Proc. The Power Electronics Show \& Conference, San Jose, CA, Oct. $7-9,1986$, pp. $294-302$.

[11] R. D. Middlebrook, "Modelling a CurrentProgrammed Boost Regulator," Proc. The Power Electronics Show \& Conference, San Jose, CA, Oct. $7-9,1986$, pp. $273-285$. 